\title{
Cochannel Interference Avoidance MAC in Wireless Cellular Networks *
}

\author{
Guowang Miao ${ }^{\ddagger}$, Ye (Geoffrey) $\mathrm{Li}^{\dagger}$, Nageen Himayat *, and Shilpa Talwar * \\ $\ddagger{ }^{\dagger}$ School of ECE, Georgia Institute of Technology \\ * Communication Technology Lab., Intel Corporation
}

\begin{abstract}
Severe cochannel interference in wireless cellular networks significantly affects users at cell edges. We propose a cost-effective cochannel interference avoidance (CIA) medium access control (MAC) scheme to improve network performance. For CIA-MAC, base stations judged as severe interferers transmit randomly and the transmission is controlled by wireless channel states to optimize the overall network performance while maintaining proportional fairness among users. Conditions for triggering CIA-MAC are derived and two simple trigger mechanisms are obtained. The CIA-MAC scheme requires low signaling overhead and only minor changes to the existing mobile systems. Simulation results show that the proposed scheme, CIAMAC, significantly outperforms traditional approaches through the avoidance of severe cochannel interference as well as the exploitation of multiuser diversity through cross-layer design.
\end{abstract}

Index Terms-interference avoidance, cross layer, distributed, medium access control, frequency reuse

\section{INTRODUCTION}

Cellular networks are becoming increasingly interference limited as more users need to share the same spectrum to achieve high-rate multimedia communications. In typical cellular systems, cochannel interference (CCI) is one of the major factors limiting capacity, especially as these systems move towards aggressive frequency reuse scenarios [1], [2]. While the overall network spectral efficiency may improve with aggressive frequency reuse, the performance of cell-edge users degrades substantially.

A commonly used method to avoid CCI is to assign different sets of channels to neighboring cells [3], [4]. A summary of different assignment methods can be found in [5]. One recent popular approach to reducing interference for cell-edge users is through fractional frequency reuse (FFR) [1], [6][9]. With FFR, full frequency reuse is applied for users at cell centers and lower frequency reuse for those at cell edges. Alternatively, CCI can be mitigated by advanced digital signal processing techniques [10]-[13]. For example, in [13], various multiuser detection and CCI suppression schemes are studied when mobile terminals (MTs) are equipped with multiple antennas. However, these techniques have high complexity and therefore, result in high implementation costs. For downlink

\footnotetext{
* This work was supported by the research gift of Intel, the U.S. Army Research Laboratory under the Collaborative Technology Alliance Program, Cooperative Agreement DAAD19-01-20-0011, and SABA Program of Motorola.

$\ddagger$ Corresponding author. Email: gwmiao@ece.gatech.edu. Address: School of Electrical and Computer Engineering Georgia Institute of Technology, Atlanta, Georgia, 30332-0250
}

transmission, CCI can be mitigated by joint pre-processing and encoding techniques among base stations (BSs) [14], [15], or avoided using cooperative scheduling among BSs [16], both of which require a lot of instantaneous information exchange. Recently, contention based schemes are developed for CCI avoidance in addition to an intracellular centralized medium access control (MAC) protocol. In [17], each MT or BS keeps on broadcasting busy-tone signals located at the mini-slot of every data frame to prevent potential interferers from transmitting, and every BS or MT listens to the minislots before transmission. This scheme effectively avoids CCI; however, fairness among users is ignored since a group of greedy users may keep on broadcasting busy-tone signals and always prevent others from transmitting.

In this paper, we develop a cost-effective cochannel interference avoidance (CIA) MAC to deal with the downlink transmission experiencing severe CCI, especially for the users at cell edges. The proposed CIA-MAC scheme maintains backward compatibility and requires only minor changes to existing BSs, while no improvement is necessary for MTs. Low overhead is added as the scheme requires only limited signaling coordination among BSs at a semi-static level. Only occasional cooperation is required when the network topology is changed and the instantaneous coordination at the packet level is not required. The proposed scheme is novel in its use of randomization by $\mathrm{BS}$ for controlling the level of interference and it provides fair transmission opportunities for the users affected by severe CCI. Although the use of randomization for collision avoidance is used extensively for uplink random access channels and wireless local-area network (LAN) systems, we are first using the principle for automatically controlling the level of downlink interference per link.

The rest of the paper is organized as follows. The system will be briefly described in Section II. In Section III, we present CIA-MAC with details. In Section IV, we address the conditions for triggering CIA-MAC and obtain two simple trigger mechanisms. The performance improvement is demonstrated through the simulation in Section V. Finally, the conclusion and future work are given in Section VI.

\section{NETWORK WITH CIA}

We only consider downlink transmission since complicated multiuser detection and CCI cancelation algorithms can be implemented at the BS for uplink CCI mitigation. The MTs at cell edges not only face the weakest signals but also suffer 


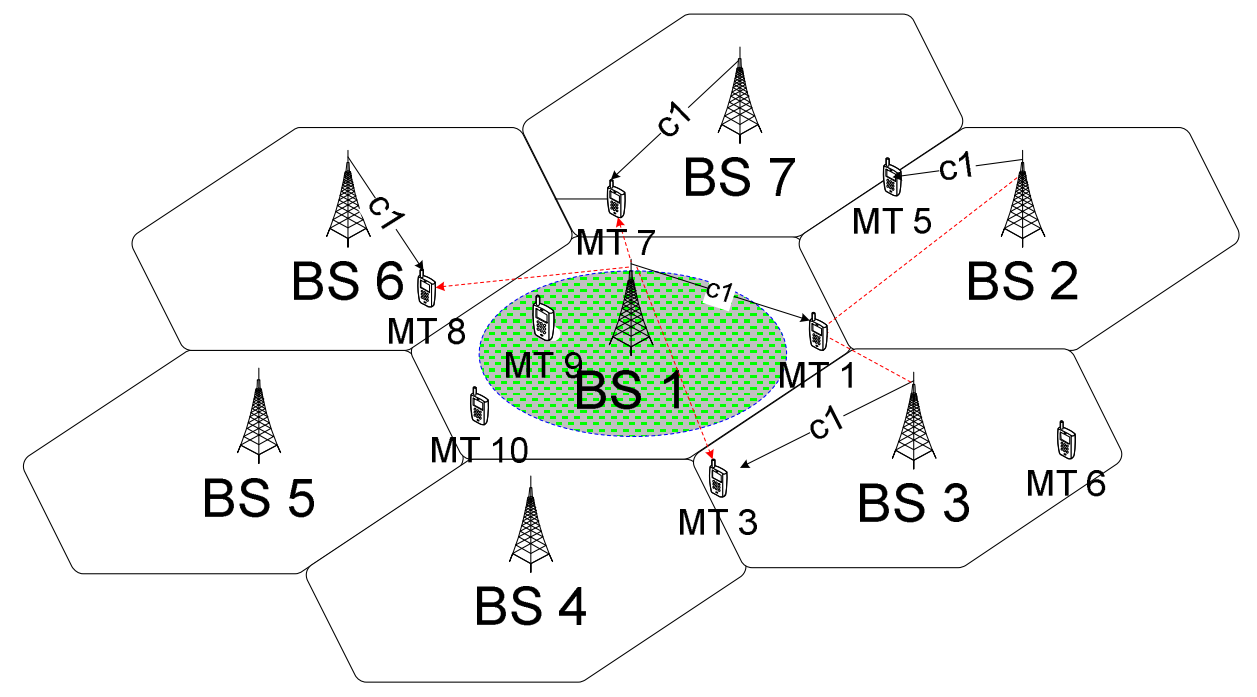

Fig. 1: Cochannel interference in cellular networks with a reuse factor of one

the largest amount of interference from neighboring cells. The CIA-MAC scheme targets the performance improvement of these users. In general, users are categorized into two classes: those experiencing no or slight CCI and those suffering from severe CCI. The first class will be scheduled by the traditional centralized MAC and the second will be first accepted by the traditional call admission control policies and then scheduled by the proposed CIA-MAC.

Each MT measures the average interference-to-carrier ratio (ICR) of neighboring BS $k$, which is defined to be

$$
I C R_{k}=\frac{E\left(h_{k} P_{k}\right)}{E(h P)},
$$

where $h$ and $P$ are the channel power gain and the transmit power of the desired link while $h_{k}$ and $P_{k}$ correspond to these of the interfering link from BS $k . E()$ is the average over a sliding window of the past data and tracks slow fading, i.e. it is a local mean and averages the effect of fast fading [5]. This definition of average will also apply in the following paragraphs.

Severe Interferer: If the ICR from neighboring $B S k$ satisfies $I C R_{k} \geq \Gamma_{m}$, the transmission of $B S k$ always causes the failure of packet reception, where $\Gamma_{m}$, called trigger, is a predetermined severe interference threshold. BS $k$ is called a severe cochannel interferer.

In Section IV, we will discuss the trigger selection and present examples.

If all BSs causing severe interference keep on transmitting, the packet receptions of the interfered MTs always fail. If BSs can collaborate, the interfering BSs may transmit in turn. However, this incurs huge signaling overhead. If there is no collaboration among BSs, we let BSs transmit randomly when identified as severe interferers. Their transmission should be managed such that the overall network performance as well as the fairness among all users are jointly optimized. Hence, besides traditional MAC, a complementary MAC is used for optimizing the randomized transmission of the severe interferers. The new complementary MAC aims at improving cellular throughput through cochannel interference avoidance, and is, therefore, called CIA-MAC.

In the following, we only consider BSs and MTs controlled by CIA-MAC and CIA-MAC will be optimized by crosslayer design to fully exploit the system capacity and multiuser diversity while maintaining fairness.

The following definitions will be used in the subsequent discussion:

- $\mathcal{B}=\{1,2, \cdots, M\}$ : set of BSs.

- $\mathcal{M}=\{1,2, \cdots, N\}=\bigcup_{i \in \mathcal{B}} \mathcal{M}_{i}$ : set of MTs. $\mathcal{M}_{i}$ is the set of MTs in the cell of BS $i$. Obviously, $\mathcal{M}_{i} \cup \mathcal{M}_{j}=$ $\emptyset, \forall i \neq j$.

- $\mathcal{E}=\left\{(i, j) \mid i \in \mathcal{B}, j \in \mathcal{M}_{i}\right\}$ : set of transmission links; $(i, j)$ denotes the link from BS $i$ to $\mathrm{MT} j$.

- $\mathcal{N}_{m}=\{(i, j) \mid \forall(i, j) \in \mathcal{E}$, transmission at link $(i, j)$ causes severe interference to MT $m\}$ : set of links whose transmission will bring severe interference to MT $m$;

- $\mathcal{T}_{(i, j)}=\{m \mid \forall m \in \mathcal{M}$, MT $m$ is severely interfered by the transmission at link $(i, j)\}$ : set of MTs severely interfered by transmission at link $(i, j)$.

Figure 1 demonstrates an example. The solid lines represent data transmission links and the dashed ones links from severe interferers. We have $\mathcal{B}=\{1,2, \cdots, 7\}, \mathcal{M}=\{1,3,5,7,8,9,10\}$, and $\mathcal{E}=\{(1,1),(2,5),(3,3),(6,8),(7,7)\}$. The transmission from BS 1 to MT 1 on channel 1 is severely interfered by transmission from BS 2 to MT 5. Meanwhile, BS 1 also causes severe CCI to MTs 3, 7, and 8. Hence, $\mathcal{N}_{1}=\{(2,5),(3,3)\}$ and $\mathcal{T}_{(1,1)}=\{3,7,8\}$.

\section{PRINCIPLE OF CIA-MAC}

In this section, we describe the principle of CIA-MAC. Figure 2 shows the MAC transmission and the frame structure. Transmission time is divided into slots with length $S$. The MAC layer of each link independently sends a MAC frame at 


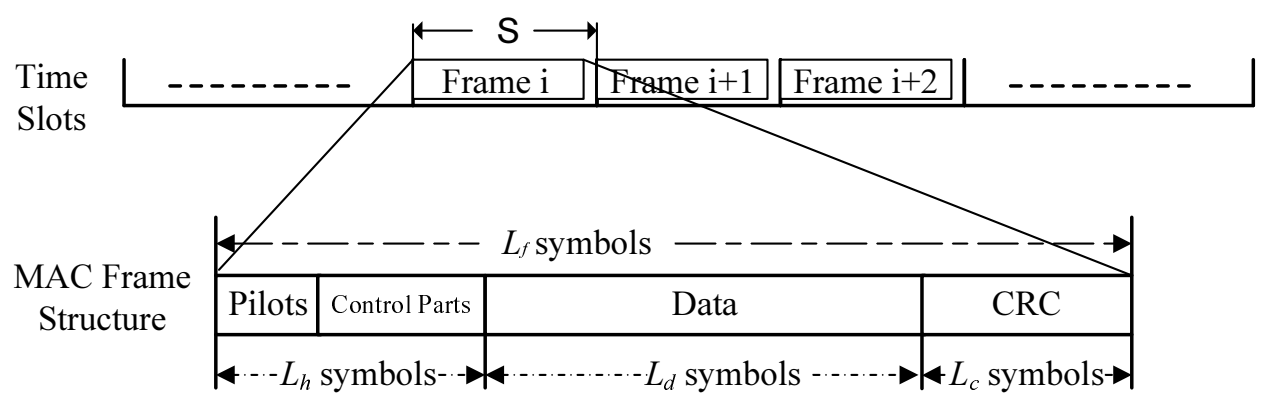

Fig. 2: MAC transmission and frame structure

the beginning of each slot with probability $p$. Complete channel state information (CSI) is known and used to determine the MAC contention and the PHY link adaptation. To obtain CSI at a transmitter [18], the CSI can be estimated through pilots at the receiver and sent to the transmitter or CSI is already available at the transmitter whenever the channel is reciprocal, such as in a time-division duplex system. Incomplete CSI results in some performance loss and the study on its impact is out of the scope of this paper. Furthermore, assume ideal cyclic redundancy check (CRC). Any error inside a frame will result in the drop of the frame. Errors are uniformly and independently distributed. Each frame has $L_{f}$ symbols, of which $L_{d}$ symbols carry data. Once the MAC layer makes a decision to transmit a frame, the frame will be continuously transmitted by the physical (PHY) layer until the frame is sent out.

In a traditional network, MAC makes transmission decision based on buffer status and quality of service (QoS) requirements and does not use PHY knowledge at all. When MAC decides to transmit, the physical channel may be in a deep fade, which wastes bandwidth and power resources. Alternatively, MAC may decide on no transmission while the channel is experiencing high gain. With cross-layer design, MAC decides whether to transmit or not according to channel information. We assume a block fading channel [19], that is, the channel state remains constant during each MAC frame. If the channel power gain at a time slot, $h$, is above a predetermined threshold $\bar{H}$, MAC sends a frame. As wireless channels are inherently random, the MAC transmission is also randomized and the threshold $\bar{H}$ determines the transmission probability. The thresholds $\bar{H}$ and the PHY transmission should be jointly optimized for all BSs subject to their power constraints. Each BS maximizes its throughput with both average power constraint $P_{a}$ and instantaneous power constraint $P_{m}$ while assuring fairness to the users in other cells.

Our previous work in decentralized optimization for multichannel random access [20], [21] will be used to optimize operations of this network. The solution in [21] maximizes the throughput product rather than the summation, i.e., the geometric average rather than the arithmetic average, of all links and hence achieves proportional fairness among all users [22]. Some analysis has been given in [22] from a game-theoretic standpoint and shows that a strategy achieving proportional fairness satisfies certain axioms of fairness [23].
With proportional fairness, the network will be operated such that no user can improve its throughput without affecting at least one user adversely. Besides fairness assurance, our solution in [21] also performs close to the globally optimal approach, which requires complete network knowledge and is too complicated to implement.

Denote the probability cumulative distribution function of channel power gain as $F(h)$ and the cardinality of $\mathcal{T}_{(i, j)}$ as $\left|\mathcal{T}_{(i, j)}\right|$. From [21], the optimal channel gain threshold for any link $(i, j) \in \mathcal{E}, \bar{H}_{(i, j)}^{*}$, that also assures proportional fairness among all CIA-MAC links, is

$$
\bar{H}_{(i, j)}^{*}=F^{-1}\left(\frac{\left|\mathcal{T}_{(i, j)}\right|}{1+\left|\mathcal{T}_{(i, j)}\right|}\right),
$$

and the corresponding transmission probability is

$$
p_{(i, j)}^{*}=\frac{1}{\left|\mathcal{T}_{(i, j)}\right|+1},
$$

where $F^{-1}(\cdot)$ denotes the inverse function of $F(\cdot)$. From (2), the optimal threshold of link $(i, j)$ depends only on $\left|\mathcal{T}_{(i, j)}\right|$, the number of MTs severely interfered by transmission at link $(i, j)$. This knowledge can be shared by the base stations of neighboring cells. $\left|\mathcal{T}_{(i, j)}\right|$ changes only when the severelyinterfered MTs have large status variations that result in the obvious changes of the ICR in (1) to go across the trigger. For example, an existing traffic session ends, a new one starts, or the movements of MTs make either a new MT severely interfered or an existing MT no longer severely interfered. These variations will trigger the update of $\left|\mathcal{T}_{(i, j)}\right|$.

In the PHY layer, consider channel inversion [24] and each BS allocates transmit power to maintain a constant received power level so that signals can be reliably detected. Once MAC decides to transmit, the transmit power is given by $P_{(i, j)}(h)=P_{r} / h$, where $P_{r}$ is the power level for reliable receiver detection. Given $\overline{\mathcal{H}}^{*}$ in (2), the received power level is optimized by [21]

$$
P_{r(i, j)}^{*}=\min \left(\frac{P_{a}}{\int_{\bar{H}_{(i, j)}^{*}}^{\infty} \frac{1}{h} f(h) \mathrm{d} h}, P_{m} \bar{H}_{(i, j)}^{*}\right) .
$$

Readers are referred to [21] for more details and more transmission optimizations.

Figure 3 illustrates the flowchart of CIA-MAC. Each MT identifies the list of neighboring BSs causing severe interference by comparing their ICRs with the trigger $\Gamma_{m}$ and reports 


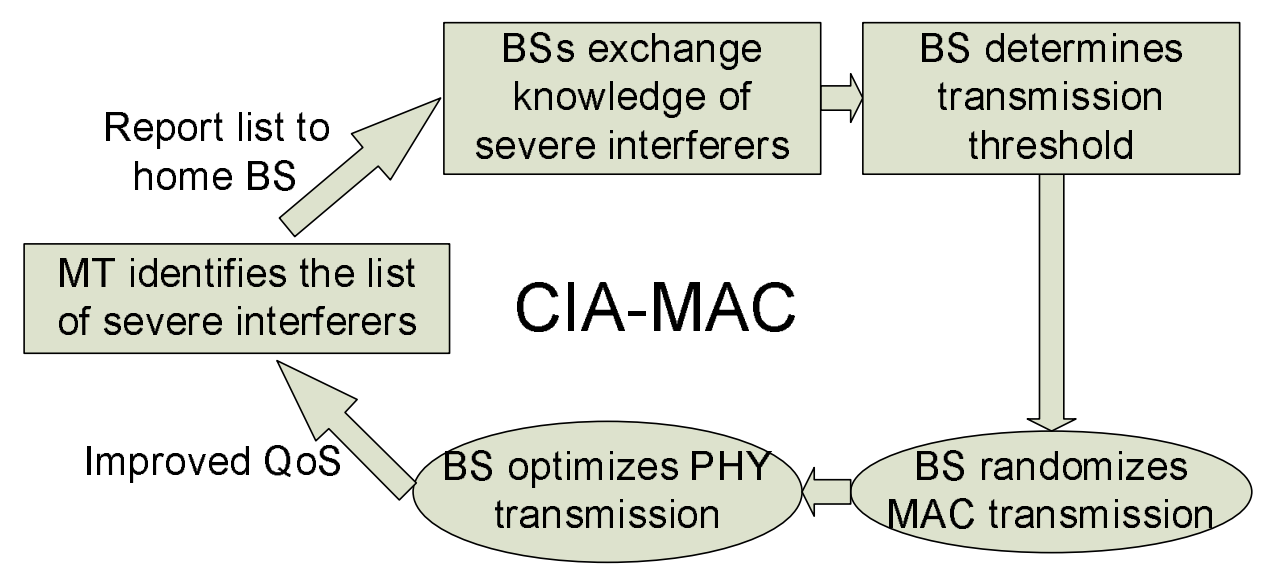

Fig. 3: CIA-MAC flowchart

the list to its home BS. The home BS communicates the list to other BSs and each BS knows the links on which it needs to randomize transmission. Then each BS determines a channel threshold $\bar{H}_{(i, j)}$ per CIA-MAC link $(i, j)$ based on the number of links affected by the transmission on that particular link. A BS transmits on a CIA-MAC link only when the channel gain on the link exceeds its channel threshold and thus randomizes the transmission. The transmission power and modulation are optimized on each link separately. As shown in Figure 3, the operations of CIA-MAC are classified into two parts. The operations in the rectangles are semi-static and take place only when the severely-interfered MTs have large status variation. With this trivial cost, the operations in the ovals automatically improve the QoS of all MTs experiencing severe interference.

\section{TRIGGer FOR CIA-MAC}

In this section, we discuss the selection of the trigger.

\section{A. Trigger selection}

Each MT measures the ICR of each neighboring BS and CIA-MAC is used at that BS when the corresponding ICR is above the trigger, $\Gamma_{m}$. Hence, the trigger determines severe interferers and relates to the performance of CIA-MAC. We will choose the trigger to maximize the throughput of the whole network rather than that of any individual link.

From [21], the throughput of link $(i, j)$ can be expressed as

$$
T_{(i, j)}=p_{(i, j)} \prod_{l \in \mathcal{N}_{j}}\left(1-p_{l}\right) \frac{R L_{d}}{S} \cdot\left(1-p_{F}\right)
$$

where $p_{l}$ is the transmission probability on link $l, \prod_{l \in \mathcal{N}_{j}}(1-$ $\left.p_{l}\right)$ is the probability that none of the severe interferers of MT $j$ transmit, $R$ is the average transmitted bits per symbol when the MAC of BS $i$ decides to transmit at link $(i, j)$ and depends on the modulation and power allocation policy, and $p_{F}$ is the frame-error rate (FER) when no severe interferers transmit.

Optimum triggers are different for MTs with different interference scenarios. Figure 1 illustrates an example. MT 3 wants to judge BS 1 . If BS 1 is judged to be a severe interferer, it will transmit with lower probability according to Equation (3). However, the impact of this variation to the packet receptions of MTs 1,7 , and 8 is unknown to MT 3 . Hence, it is difficult for each MT to evaluate the variation of the overall network throughput that results from the judgement of severe interferers. Even assume that these knowledge can be shared, different MTs have different interfering scenarios, and judging severe interferers and exchanging signaling would be daunting tasks across the whole network.

We will get one trigger for all to simplify the calculations. Consider a network in which each MT is severely interfered by $K$ neighboring BSs on average. The BS of each MT also brings severe CCI to $K$ MTs in the neighboring cells on average. Empirical values can be assigned to $K$, e.g. $K=3$ is a good choice based on our simulation observations for a reuse one network. From equation (3), the transmission probability of each BS is $\frac{1}{1+K}$ and the throughput is

$$
\begin{aligned}
T & =\frac{1}{1+K}\left(1-\frac{1}{1+K}\right)^{K} \frac{R L_{d}}{S}\left(1-p_{F}\right) \\
& =\frac{K^{K}}{(1+K)^{K+1}}\left(1-p_{b}\right)^{R L_{d}} \frac{R L_{d}}{S},
\end{aligned}
$$

where the frame error rate is approximated by $p_{F}=1-$ $\left(1-p_{b}\right)^{L_{d} R}$ according to the assumption of uniform and independent error distribution in Section III. The bit-error rate (BER) is approximated by $p_{b}=P_{e}(\eta)$, where $\eta$ is the average signal-to-noise ratio (SNR) and the BER function $P_{e}()$ depends on the modulation and coding. For example, the BER for coherently detected m-ary quadrature amplitude modulation (M-QAM) with Gray mapping over an additive white Gaussian noise channel can be well approximated by [25]

$$
P_{e}(\eta) \approx 0.2 \exp \left(-\frac{1.5 G_{c} \eta}{M-1}\right),
$$

where $G_{c}$ is the coding gain and $M$ is the modulation order.

With the traditional MAC, all BSs keep on transmitting. Each link has throughput

$$
\widehat{T}=\frac{L_{d} \widehat{R}}{S} \cdot\left(1-\widehat{p}_{F}\right)=\frac{L_{d} \widehat{R}}{S} \cdot\left(1-\widehat{p}_{b}\right)^{L_{d} \widehat{R}},
$$

where $\widehat{p}_{F}$ and $\widehat{p}_{b}$ are the average frame and bit error rates, and $\widehat{R}$ is the average number of bits transmitted per symbol in this 


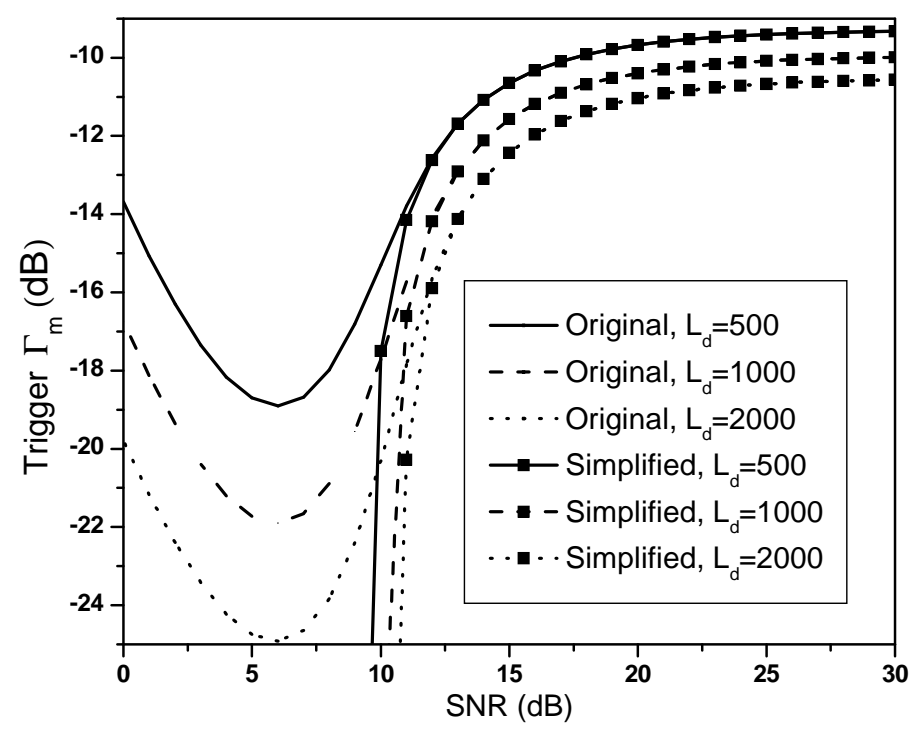

Fig. 4: Trigger of severe cochannel interferer

mode. $\widehat{p}_{b}, \widehat{p}_{F}$, and $\widehat{R}$ are different from those in (6) since BSs have different transmission durations and signal receptions are with different interference scenarios, which result in different power and modulation allocation approaches. CIA-MAC is triggered when it achieves better throughput, i.e. $T>\widehat{T}$, or

$$
\frac{K^{K}}{(1+K)^{K+1}}\left(1-p_{b}\right)^{L_{d} R} \frac{L_{d} R}{S}>\left(1-\widehat{p}_{b}\right)^{L_{d} \widehat{R}} \frac{L_{d} \widehat{R}}{S},
$$

Then we have

$$
\widehat{p}_{b}>1-\left(\frac{K^{K}}{(1+K)^{K+1}}\left(1-p_{b}\right)^{L_{d} R} \frac{R}{\widehat{R}}\right)^{\frac{1}{L_{d} \widehat{R}}} .
$$

Since $\widehat{p}_{b}=P_{e}(\widehat{\eta})$, where $\widehat{\eta}$ is the average signal-tointerference-plus-noise ratio (SINR),

$$
\widehat{\eta}<P_{e}^{-1}\left[1-\left(\frac{K^{K}}{(1+K)^{K+1}}\left(1-p_{b}\right)^{L_{d} R} \frac{R}{\widehat{R}}\right)^{\frac{1}{L_{d} \hat{R}}}\right] .
$$

$S I N R$ and $I C R$ follow the relationship

$$
I C R=\frac{1}{\widehat{\eta}}-\frac{1}{\eta} \text {. }
$$

The trigger $\Gamma_{m}$ follows immediately

$$
\Gamma_{m}=\frac{1}{P_{e}^{-1}\left[1-\left(\frac{K^{K}}{(1+K)^{K+1}}\left(1-P_{e}(\eta)\right)^{L_{d} R} \frac{R}{\widehat{R}}\right)^{\frac{1}{L_{d} \hat{R}}}\right]}-\frac{1}{\eta} .
$$

$\Gamma_{m}$ depends on $S N R$ and BER function, both of which are known to each MT. Hence, $\Gamma_{m}$ can be easily calculated for judgement of severe interferers.

For fixed modulation, $R=\widehat{R}$. The trigger is

$$
\Gamma_{m}=\frac{1}{P_{e}^{-1}\left[1-\left(\frac{K^{K}}{(1+K)^{K+1}}\right)^{\frac{1}{L_{d}^{R}}}\left(1-P_{e}(\eta)\right)\right]}-\frac{1}{\eta} \text {. }
$$

For normal data transmission, $S N R$ is high and $P_{e}(\eta) \ll 1$, thus (14) is further simplified to be

$$
\Gamma_{m}=\frac{1}{P_{e}^{-1}\left[1-\left(\frac{K^{K}}{(1+K)^{K+1}}\right)^{\frac{1}{L_{d} R}}\right]}-\frac{1}{\eta} .
$$

\section{B. An alternate trigger mechanism using location knowledge}

In the flowchart of Figure 3, each MT determines the list of severe interferers and reports the list to the home BS, which requires additional improvement of MTs. In the following, we show how to enable BSs to determine the severe interferers to avoid the necessity of MT improvement.

We assume that BSs have the position knowledge of MTs in both the home cells and the neighboring cells. Note that a large quantities of positioning techniques have been proposed in cellular networks [26]-[29]. Hence, it is practical to obtain the position knowledge of each MT and this knowledge can be shared among neighboring BSs. Besides, assume that each BS knows the average received signal power at a desired MT, which can be obtained through feedback or observation of link power control. This knowledge will also be shared among neighboring BSs.

We have shown that the optimal threshold (2) for each BS depends on the number of MTs severely interfered by its transmission. This number can be obtained through cooperation among BSs. A BS located at coordinate $\left(x_{b}, y_{b}\right)$ needs to determine whether it brings severe interference to the neighboring-cell MT at coordinate $\left(x_{m}, y_{m}\right)$. The distance between them is

$$
d_{I}=\sqrt{\left(x_{b}-x_{m}\right)^{2}+\left(y_{b}-y_{m}\right)^{2}},
$$

which results in path loss $L\left(d_{I}\right)$. The average received signal power at the MT is $P_{s}$ while the interfering BS has the average transmit power $P_{I}$. According to (1), the average interference to carrier ratio is

$$
I C R=\frac{P_{I} h_{I}}{P_{s}},
$$


where $h_{I}$ is the average channel power gain of this interfering link. However, $h_{I}$ is unknown to the interfering BS and needs to be estimated. Radio propagation is characterized by three nearly independent phenomena: path loss variation with distance, slow log-normal shadowing, and fast multipath fading [5]. Similar to the ICR in (1), $h_{I}$ tracks slow fading, i.e. it is a local mean and averages the effect of fast multipath fading. Hence, we consider only the path loss and shadowing. Shadow represents the error between the actual and estimated path loss [5]. While the estimated path loss is determined by the radio path distance $d$, the shadowing/estimation error has been observed to be nearly independent of $d$ and we assume the independence. Hence, we model the estimated average interference channel gain by two parts: the estimated path loss determined by path loss model $L(d)$ and the estimation error determined by the shadowing model. Shadows are generally modeled as being log-normally distributed and $10 \log _{10}\left(h_{I}\right)$ has normal distribution with mean $-10 \log _{10}(L(d))$ and standard deviation $\sigma$, where $\sigma$ is independent of the radio path length $d$ and typically ranges from 5 to $12 \mathrm{~dB}$ [5]. To ensure a detection probability $\beta$ of severe interferers, the BS determines that the MT is severely interfered when the probability of severe interference is above $\beta$, i.e.

$$
\operatorname{Prob}[\text { severe interference }]=\operatorname{Prob}\left[I C R \geq \Gamma_{m}\right] \geq \beta,
$$

which is equivalent to

$$
\operatorname{Prob}\left[10 \log _{10}\left(h_{I}\right) \geq 10 \log _{10}\left(\frac{\Gamma_{m} P_{s}}{P_{I}}\right)\right] \geq \beta \text {. }
$$

Solving (19) yields the detector of severe interference as follows

$$
\frac{P_{I}}{L\left(d_{I}\right) P_{s}} \geq \Gamma_{m} 10^{-\frac{\sigma Q^{-1}(\beta)}{10}} \triangleq \Gamma_{b},
$$

where $Q(x)$ is the right-tail probability of the standard normal distribution, that is

$$
Q(x)=\int_{x}^{\infty} \frac{1}{\sqrt{2 \pi}} e^{-\frac{t^{2}}{2}} d t .
$$

With (20), each BS can detect how many MTs are severely interfered and determine its transmission probability (3) as well as the threshold (2). In this case, no MT improvement is necessary for the functioning of CIA-MAC. Note that the selection of $\beta$ determines how pessimistically or optimistically a severe interferer is judged.

\section{NUMERICAL AND Simulation RESUlts}

In this section, we show the relationship between the trigger and SNR, verify the effectiveness of the trigger, and demonstrate the performance of CIA-MAC in a cellular network through comparison with the traditional MAC and a static FFR approach.

In the cellular system simulated, the radius of each cell is $2 \mathrm{~km}$ and no sectoring is used. The thermal noise power is $-104 \mathrm{dBm}$ over the whole bandwidth. The carrier frequency is $900 \mathrm{MHz}$. BSs are 100 meters high with $8.2 \mathrm{~dB}$ antenna gain while MTs have height 1.5 meters with $2.2 \mathrm{~dB}$ antenna gain. Path loss is given by the urban-area Hata-Okumura model. Log-normal shadowing and Rayleigh fading are applied. Each
MAC frame consists of 1000 symbols, in which 900 carry payload.

\section{A. Relationship of trigger and SNR}

Considering uncoded 4-QAM modulation, the relationship between the trigger $\Gamma_{m}$ and $S N R$ when $L_{d}$ has different values is illustrated by Figure 4 . The amount of bits transmitted per MAC frame varies and is usually very large to fully exploit link capacity. For example, in 802.16e [1], each frame has a maximum length of 2048 bytes of payload followed by one CRC verification, i.e. 16384 bits of payload per frame. In high-speed downlink packet access (HSDPA) transmission of universal mobile telecommunications system (UMTS) [2], the size of a transport block followed by one CRC verification ranges widely from 15890 bits to 204000 bits. In general, we assume large $L_{d} R$ and illustrate the cases when $L_{d}$ is 500 , 1000 , and 2000 respectively. The curves without markers are calculated through (14) while those with markers through (15). Figure 4 clearly shows that (15) is a good approximation of the threshold for high SNR. In the high-SNR region, the receiver can bear higher interference when signal power increases, yielding increasing trend of the curve. In the low-SNR region, when SNR goes lower, that is, noise power goes higher, interference needs to have stronger power to impact more on frame reception than noise. This indicates the increasing trend of the curve as SNR goes lower in the low-SNR region. This also indicates that in the noise-dominated region, it is better to ignore interference, as suppressing it will not provide much advantage.

\section{B. Effect of the trigger}

Consider a simplified cellular network. Each BS serves one user on a channel. Compare the performance of CIA-MAC and the traditional MAC that keeps on transmitting. All MTs are located at the same distance away from their corresponding home BSs and the distance goes from 0 to the cell radius. Each MT is severely interfered by one neighboring BS and each BS causes severe CCI to a MT in a neighboring cell. Assume uncoded 4-QAM modulation and a transmit power of $43 \mathrm{dBm}$. Figure 5(a) shows the relationship between the ICR and the trigger when the network has a reuse factor of either one or three. Figure 5(b) shows the throughput of both CIA-MAC and the traditional MAC. Since the trigger depends on signal and noise powers but not interference, it is independent of the network reuse type, which determines interference environment. As a MT moves closer to a cell edge, the trigger decreases because of decreasing signal power while the ICR increases because of growing interference power. From Figure 5, CIA-MAC is triggered when the ICR is above the trigger and achieves better throughput than the traditional MAC through interference avoidance. The network performance is improved for MTs experiencing severe interference without equipping them with the ability to mitigate interference. We also see that the performance improvement of CIA-MAC is low when the reuse factor is three and will be even more trivial with higher reuse factors. This is because a higher reuse factor results in less severe interference and 


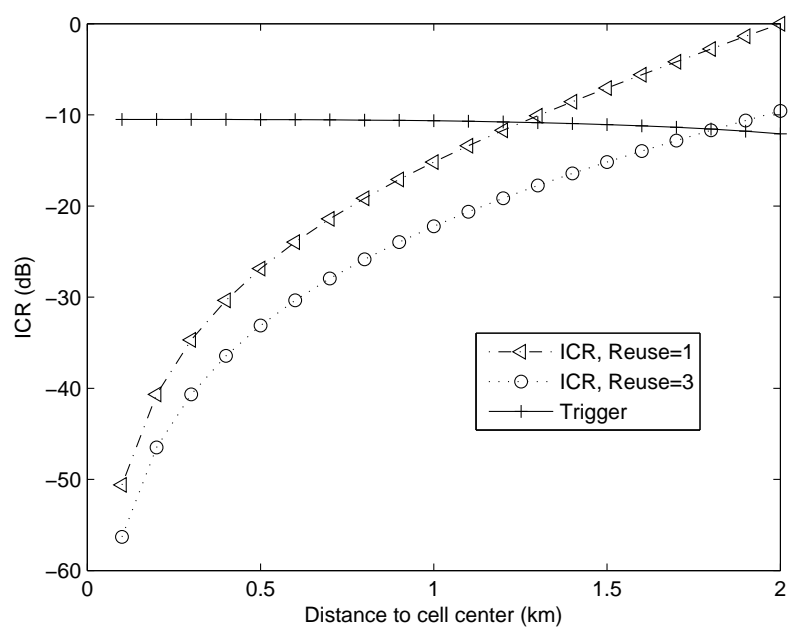

(a)

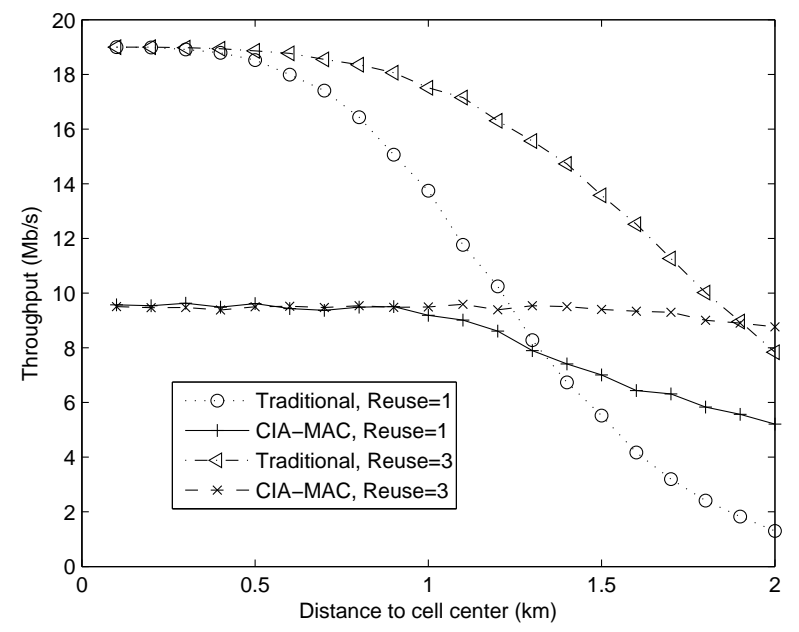

(b)

Fig. 5: Trigger effect.

interference avoidance is less desired. Therefore, CIA-MAC is good for networks with low reuse factors. Note that network deployment with lower frequency reuse factors is a popular trend in the next-generation communication systems [1], [2] for achieving higher spectrum efficiency and reducing network deployment cost.

\section{Network performance improvement}

In this section, we demonstrate the performance of CIAMAC in a nineteen-cell cellular network. Each BS serves one user on a channel. Users are randomly dropped and uniformly distributed in each cell for each simulation trial.

CIA-MAC is implemented either with or without crosslayer design. The one with cross-layer design follows what we have discussed in this paper and transmission happens only when channel power gain is above the threshold in Equation (2). For CIA-MAC without cross-layer design, each BS transmits randomly with probability given by Equation (3) and independently of channel states. We implement both trigger mechanisms and in the second one, the detection probability is set to be 0.9 . We compare CIA-MAC with the traditional MAC and a static FFR and the overall system bandwidth is the same for all of them. The network has a reuse factor of one for both CIA-MAC and the traditional MAC. The traditional MAC keeps all BSs transmitting and users experience interference from all neighboring BSs. The static FFR reduces cell-edge interference through low frequency reuse at cell edges [1], [6]-[9]. Figure 6 illustrates the network frequency deployment of FFR in our simulation. The radius of each cell is $r_{1}=2 \mathrm{~km}$ and the cell-center users, located within $r_{2}=2 r_{1} / 3$ from the BS, will transmit over the whole frequency band. For cell-edge communications, the whole frequency band is equally divided into three subbands, $f_{1}$, $f_{2}$, and $f_{3}$, and users at cell edges are assigned one of them according to the frequency deployment in Figure 6. There are two interfering circumstances for FFR users. Cell-center users experience interference from all neighboring BSs. Neighboring BSs that use only one of the three subbands produce $1 / 3$ interference power since interference power distributes only in $1 / 3$ of the whole signal bandwidth. Cell-edge users experience interference from both the first-tier cells that are using the whole bandwidth and the second-tier cells that are using the same frequency subband as the center cell.

Note that the interference in Cell 1 that comes from the first-tier cells dominates the interference power and determines the performance of both the traditional MAC and FFR. Furthermore, the performance of CIA-MAC depends on the interference environments in both the first-tier and second-tier cells according to Equations (2) and (5). Hence, the performances of CIA-MAC, traditional MAC, and FFR in Cell 1 are representative for their corresponding per-cell performance in general multi-cell cellular networks and we focus on the performance of cell 1. In Figures 7, 8, and 9, we compare the SINR and throughput of different schemes in Cell 1. Either uncoded 4-QAM or coded 4-QAM with different coding gains is used. The coding gain can be obtained through exploitation of receiver diversity or channel coding. In each trial, all BSs allocate the transmit power to maintain a constant received signal power level, i.e. keep a fixed SNR.

In Figure 7, we fix the SNR to be $12 \mathrm{~dB}$ and compare the cumulative distribution functions (CDFs) of average SINR of each trial. It includes statistics over 50, 000 trials. SINR is the equivalent value after decoding when coded 4-QAM is used. For reference, the relationship between FER and SINR is also plotted with a bold curve. Observing the FER curve, when SINR is lower than $10 \mathrm{~dB}$, most decoded frames have at least one bit in error and do not pass CRC, resulting in transmission outage. In Figure 7, we compare the schemes with either uncoded 4-QAM or coded 4-QAM that has $8 \mathrm{~dB}$ coding gain. For CIA-MAC, only SINRs when no severe interferes transmit are averaged to produce the CDF curves. Curves with legend $C I A-M A C_{b}$ correspond to the performance of 
CIA-MAC when severe interferers are determined by the BSs according to (20). We can see that $C I A-M A C_{b}$ performs closely to $C I A-M A C$ in both cases and while significantly reducing the improvement cost, BS judgement is effective in detecting severe interferers using position knowledge. Without coding, the traditional MAC suffers strong interference and the average SINRs of all simulation trials falls far below $10 d B$. In this case, the network is completely in outage. With FFR, the average SINRs of all trials are significantly improved. However, interference from neighboring cells still affects the SINRs and most SINRs are less than $10 \mathrm{~dB}$ since the target SNR is only $12 \mathrm{~dB}$. Amazingly, CIA-MAC has better SINR distribution than FFR, even the one without crosslayer design. This is because we only average SINRs when no severe interferes transmit, i.e. when transmission succeeds in CIA-MAC. Furthermore, CIA-MAC with cross-layer design achieves very high average SINR. This is because the BS in Cell 1 also brings severe interference to many MTs in the neighboring cells, e.g. an average of 3.52 MTs in the simulation, resulting in a very high threshold in (2) and thus high average SINR. Now observe the performance of schemes with coded 4-QAM that has $8 \mathrm{~dB}$ coding gain. We can see that the SINR performance of both FFR and the traditional MAC are improved by around $8 \mathrm{~dB}$. Since MTs can mitigate a large amount of interference with coding, CIA-MAC judges much less severe interferers, e.g. 0.2 on average in our simulation. Hence, CIA-MAC finds no severe interferers in most cases and BSs simply keep on transmitting, as what the traditional MAC does. However, CIA-MAC still outperforms the traditional MAC because of avoidance of severe interference whenever it exists. Note that with high coding gain, CIA-MAC with cross-layer design has lower average SINR as compared with uncoded cases. This is because with $8 \mathrm{~dB}$ coding gain, only few severely interfered MTs are judged in the most trials and the BS is allowed to transmit in most channel conditions rather than very good ones. However, SINR performance does not solely determine the network performance, which also relate to spectral reuse efficiency, transmission probability, and so on. For example, compare the performance of CIA-MAC with cross-layer design when either coded or uncoded 4-QAM is used. The SINR performance with uncoded 4-QAM even outperforms the coded one with $8 \mathrm{~dB}$ coding gain. However, it is at the price of extremely low probability to transmit with only peak channel conditions. With high coding gain, BSs produce interference bearable to neighboring-cell MTs and are allowed to transmit at high probability. Both SINR and transmission probability impact the throughput, which is the performance we desire to improve. In the following, we further compare the network throughput.

In Figure 8, we compare the average throughput when the target SNR has different values. For each SNR, the throughput is the average over 50,000 trials. From the figure, for a system with uncoded 4-QAM, the traditional MAC has almost no throughput improvement when SNR is increased. This is because with increased SNR, transmit powers of neighboring $\mathrm{BSs}$ also increase, resulting in stronger interference and trivial SINR improvement. This indicates the necessity of tackling CCI for this highly aggressive frequency reuse scenario.
Through frequency reuse at cell edges, FFR successfully reduces interference. With FFR, the average SINR improves as SNR increases and thus higher throughput is obtained. However, in the low-SNR region, interference significantly affects frame reception due to weak signal power and even with frequency reuse at cell edges, FFR still suffers from neighboring-cell interference. We can see that CIA-MAC schemes significantly outperforms all other schemes. This is due to the intelligent interference avoidance and full frequency reuse in CIA-MAC. With cross-layer design, transmission happens only with high channel power gain, which further improves throughput as compared with the one without crosslayer design. Note that the CIA-MAC with BS determining severe interferers performs closely to the one with MT, indicating good detection capability of BSs. Similar to the traditional MAC, CIA-MAC also suffers a saturation effect in the highSNR region, i.e. increasing transmit power does not necessarily result in improved throughput because of proportionally increased interference power. With $8 \mathrm{~dB}$ coding gain, mobiles can mitigate a large portion of interference and all schemes have significant performance enhancement. We note that our proposed schemes, both with and without cross-layer design, still outperform the traditional MAC comprehensively due to the intelligent recognition of severe interferers for interference avoidance. The throughput of FFR increases with SNR in the low-SNR region due to increased SINR. In the high-SNR region, FFR throughput does no improve with SNR since all frames have been correctly received. However, in the highSNR region, FFR performs not as well as the proposed CIAMAC due to its low spectrum reuse efficiency at cell edges. Figure 9 further demonstrates this point. In Figure 9, each BS allocates power to maintain a $9 \mathrm{~dB}$ received $\mathrm{SNR}$ and the coding gains of all MTs are increased from $0 \mathrm{~dB}$ to $15 \mathrm{~dB}$. We observe that CIA-MAC always outperforms the traditional MAC. With higher and higher coding gain, interference has less and less impact on frame reception and thus fewer and fewer severe interferers are judged. Hence, with high coding gain, the performance of CIA-MAC and traditional MAC tend to be the same. The static FFR suffers performance loss for low frequency-reuse efficiency at cell edges in the high-codinggain region. Dynamic FFR schemes [7]-[9] can be used to further improve frequency-reuse efficiency at the cost of higher network deployment complexity. However, we should note that CIA-MAC improves the network performance in the most cost-effective way and even the static FFR implemented here requires much higher deployment cost than CIA-MAC.

\section{CONCLUSiOns And Future Work}

This paper provides a low-cost solution to improve the performance for cell-edge MTs that are experiencing severe CCI in wireless cellular networks. The proposed CIA-MAC requires semi-static information exchange among BSs and automatically randomizes transmission to improve QoS for severely interfered MTs. The principle for triggering CIAMAC is investigated and two simple trigger mechanisms are described. The proposed scheme significantly improves communication performance for MTs experiencing severe CCI 


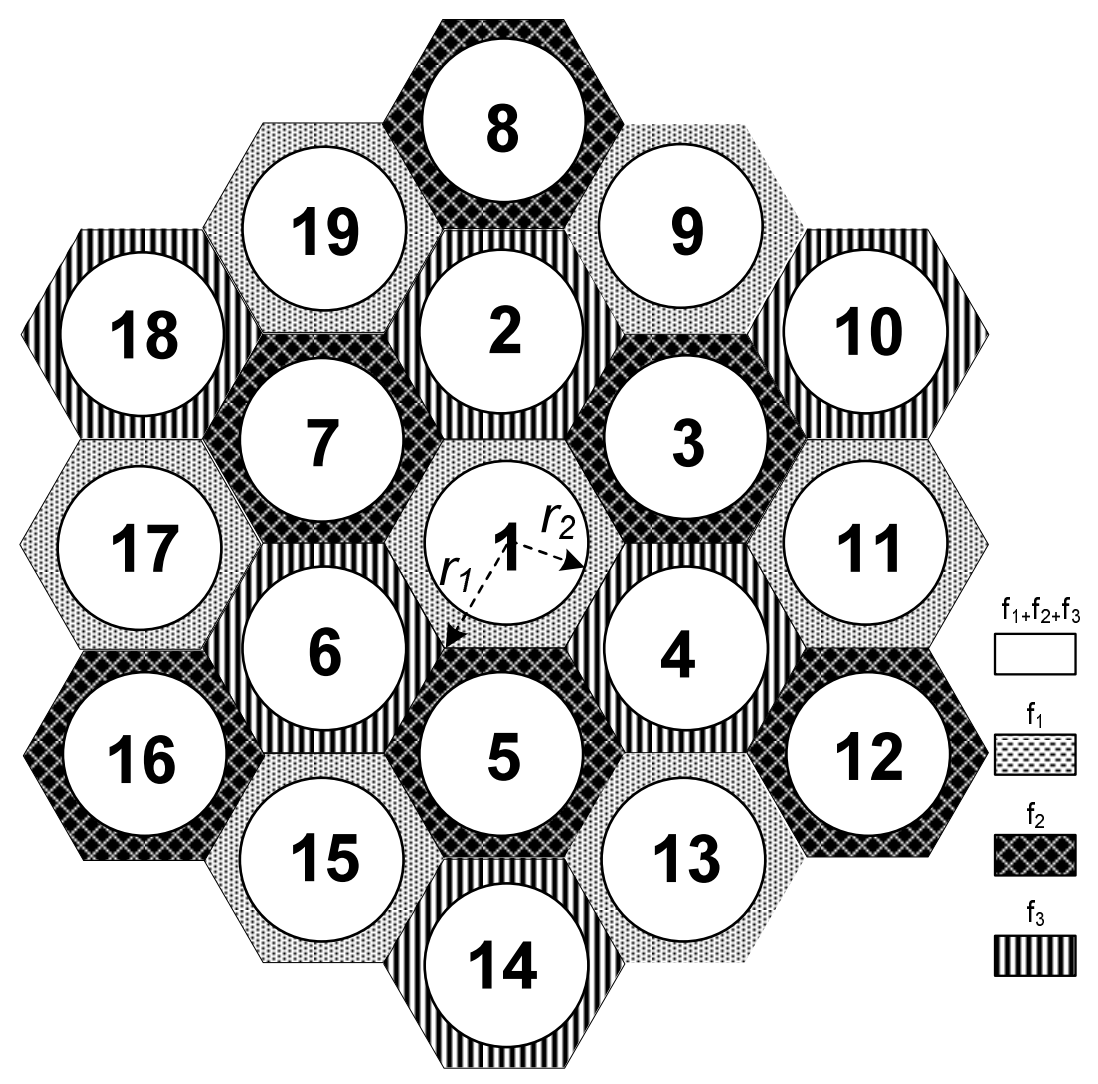

Fig. 6: Cellular networks with fractional frequency reuse

because of intelligent recognition of severe interferers and the corresponding interference avoidance.

For simplicity, we did not consider traffic characteristics, which influence MAC buffer status and thus its transmission probability. The thresholds will be redesigned to incorporate traffic characteristics in our future research. Besides, reservation based CIA-MAC as well as multi-channel extensions may be designed to further enhance network performance.

\section{REFERENCES}

[1] IEEE Std 802.16e-2005, "IEEE Standard for Local and Metropolitan Area Networks - Part 16: Air Interface for Fixed and Mobile Broadband Wireless Access Systems - Amendment 2: Physical and Medium Access Control Layers for Combined Fixed and Mobile Operation in Licensed Bands," Feb., 2006.

[2] 3GPP TS 25.321, "3rd Generation Partnership Project; Technical Specification Group Radio Access Network; Medium Access Control (MAC) Protocol Specification ," v7.5.0, July, 2007.

[3] R. Prakash, N.Shivaratri, and M. Singhal, "Distributed dynamic faulttolerant channel allocation for mobile computing," IEEE Trans. Veh. Tech., vol. 48, no. 6, pp. 1874-1888, Nov. 1999.

[4] G. Cao and M. Singhal, "An adaptive distributed channel allocation strategy for mobile cellular networks," J. Parallel and Dist. Comput., vol. 60, no. 4, pp. 451-473, 2000.

[5] G. L. Stüber, Principles of Mobile Communication, Kluwer Academic Publishers, Jan, 2001.

[6] K. Begain, G. I. Rozsa, A. Pfening, and M. Telek, " Performance analysis of GSM networks with intelligent underlay-overlay," in Proc. 7th Int. Symp. on Comp. and Commun. (ISCC 2002), 2002, pp. 135-141.

[7] M. C. Necker, "Coordinated fractional frequency reuse," in Proc. 10th ACM Symp. on Mod., Anal., and Sim. of wireless and Mob. Syst., 2007, pp. 296-305.

[8] 3GPP TSG-RAN R1-050764, "Inter-cell interference handling for EUTRA,” Ericsson, Sep. 2005.
[9] 3GPP TSG RAN R1-050841, "Further analysis of soft frequency reuse scheme," Huawei, Aug. 2005.

[10] J. G. Andrews, "Interference cancellation for cellular systems: a contemporary overview," IEEE Wireless Commun., vol. 12, no. 2, pp. 19-29, Apr. 2005.

[11] H. Schoeneich and P. A. Hoeher, "Single antenna interference cancellation: iterative semi-blind algorithm and performance bound for joint maximum-likelihood interference cancellation," in Proc. IEEE Global Commun. Conf. 2003, San Francisco, CA, Dec. 2003, pp. 1716-1720.

[12] J. Li, B. K. Letaief, and Z. Cao, "Co-channel interference cancellation for space-time coded OFDM systems," IEEE Trans. Wireless Commun., vol. 2, no. 1, pp. 41-49, Jan. 2003.

[13] H. Dai, A. F. Molisch, and H. V. Poor, "Downlink capacity of interference-limited MIMO systems with joint detection," IEEE Trans. Wireless Commun., vol. 3, no. 2, pp. 442-453, Mar. 2004.

[14] S. Shamai and B. M. Zaidel, "Enhancing the cellular downlink capacity via co-processing at the transmitting end," in Proc. Conf. Veh. Tech. Spring (VTC 2005). vol. 3, no. 3, May 2001, pp. 1745-1749.

[15] H. Zhang and H. Dai, "Cochannel interference mitigation and cooperative processing in downlink multicell multiuser MIMO networks," EURASIP J. Wireless Commun. and Networking, pp 222-235, Feb. 2004.

[16] W. Choi and J. G. Andrews, "Base station cooperatively scheduled transmission in a cellular MIMO TDMA system," in Proc. 40th Annual Conf. Inf. Sci. Sys., Mar. 2006, pp. 105-110.

[17] P. E. Omiyi and H. Haas, "Improving time-slot allocation in $4^{\text {th }}$ generation OFDM/TDMA TDD radio access networks with innovative channel-sensing," in Proc. IEEE Int. Conf. Commun (ICC' 2004), no. 6, June 2004, pp. 3133-3137.

[18] T. Keller and L. Hanzo, "Adaptive multicarrier modulation: a convenient framework for timefrequency processing in wireless communications," in Proc. of the IEEE, no 88, May, 2000, pp. 611-640.

[19] R. J. McEliece and W. E. Stark, "Channels with block interference," IEEE Trans. Inf. Theory, vol. 30, no. 1, pp. 44-53, Jan 1984.

[20] G. W. Miao, Y. (G.) Li, and A. Swami, "Decentralized cross-layer optimization for multichannel aloha wireless networks," in IEEE Global Commun. Conf. 2007, Washington, DC, Nov. 2007, pp. 4456-4460.

[21] G. W. Miao, Y. (G.) Li, and A. Swami, "Decentralized optimization for multichannel random access," to appear in IEEE Trans. Commun. 


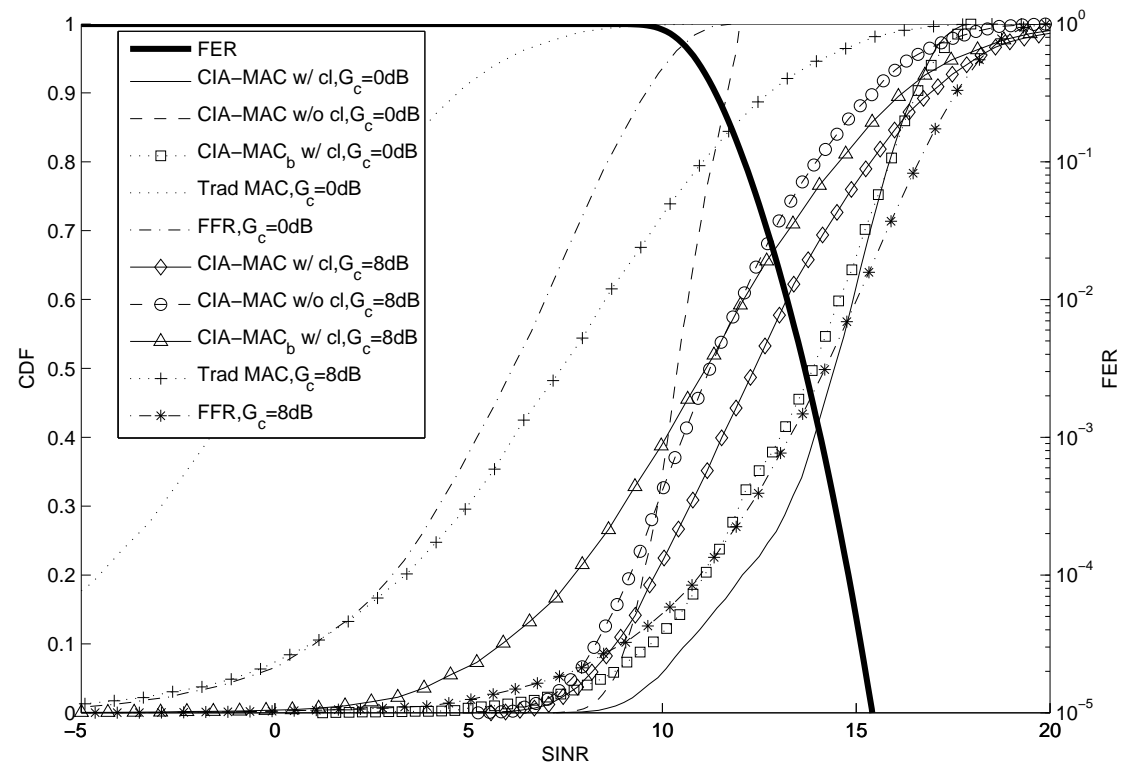

Fig. 7: Cumulative distribution function of SINR.

[22] R. Mazumdar, L. G. Mason, and C. Douligieris, "Fairness in network optimal flow control: optimality of product forms," IEEE Trans. Commun., vol 39, no. 5, pp. 775-782, May 1991.

[23] P. D. Straffin, "Game theory and strategy," Mathematical Association of America , 1993.

[24] A. J. Goldsmith and P. P. Varaiya, "Capacity of fading channels with channel side information," IEEE Trans. Inf. Theory, vol 43, no. 6, pp. 1986-1992, July 1997.

[25] A. J. Goldsmith and S. G. Chua, "Wireless Communications," Cambridge University Press, Aug. 2005.

[26] M. Hellebrandt, R. Mathar, and M. Scheibenbogen, "Estimating position and velocity of mobiles in a cellular radio network," IEEE Trans. Veh. Tech., vol. 46, no. 1, pp. 65-71, Feb. 1997.

[27] C. Drane, M. Macnaughtan, and C. Scott, "Positioning GSM telephones," IEEE Commun. Mag., vol. 36, no. 4, pp. 45-54, Apr. 1998.

[28] M. Hellebrandt and R. Mathar "Location tracking of mobiles in cellular radio networks martin," IEEE Trans. Veh. Tech., vol. 48, no. 5, pp. 15581862, Sep. 1999.

[29] G. Sun, J. Chen, W. Guo, and K.J.R. Liu, "Signal processing techniques in network-aided positioning: a survey of state-of-the-art positioning designs," IEEE Sig. Processing Mag., vol. 22, no. 4, pp. 12-24, July, 2005. 


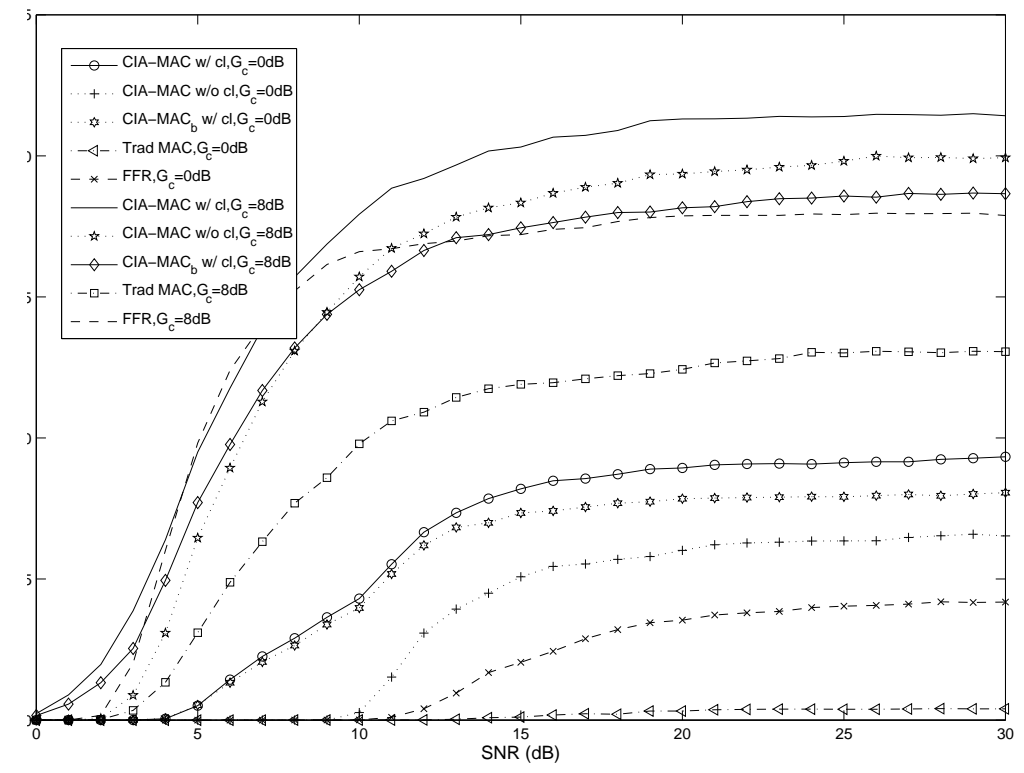

Fig. 8: Throughput comparison

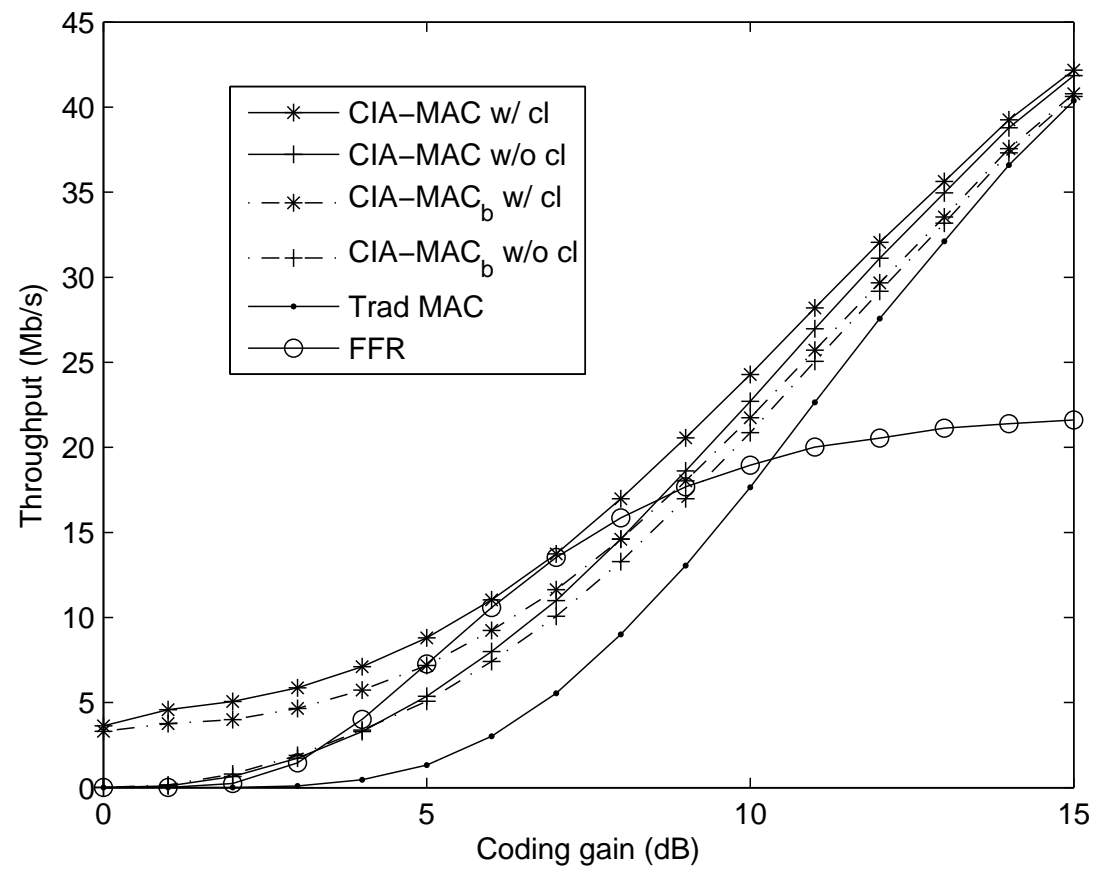

Fig. 9: Throughput comparison $(\mathrm{SNR}=9 \mathrm{~dB})$ 